\title{
Yes, we CAM! Oder: Europa, wir kommen!
}

\author{
Dieter Melchart \\ Zentrum für naturheilkundliche Forschung der II. Medizinischen Klinik und Poliklinik, Technische Universität München, Deutschland
}

Gratulation an die Initiativgruppen! Viele Jahre bemühten sich insbesondere die Wiener Arbeitsgruppe der Internationalen Akademie für Ganzheitsmedizin und einige «Aufrechte» aus Berlin, Bern und München um einen organisierten Dialog in Richtung Europa. Erklärtes Ziel war die Integration der Komplementär- und Alternativmedizin (CAM) in das europäische Forschungsförderprogramm FP7. Durch Ausdauer, Fleiß und Unterstützung der Initiativgruppen nicht zuletzt durch den Einsatz von Wolfgang Weidenhammer (mit besonderer Unterstützung durch Benno Brinkhaus) für die schriftliche Beantragung und Koordination der Forschungsanträge - konnte der bisher größte Erfolg der CAMForschung in Europa, nämlich eine offizielle Aufnahme in das EU-Forschungsprogramm, verwirklicht werden.

Im Januar 2010 war es dann endlich so weit: Die Kick-offVeranstaltung für das CAMbrella-Projekt konnte in München - unter Leitung des Koordinators Wolfgang Weidenhammer vom Zentrum für naturheilkundliche Forschung des Klinikums rechts der Isar - durchgeführt werden.

CAMbrella betreibt als sogenanntes Koordinierungsprojekt keine eigene Forschung, sondern soll ein Netzwerk verschiedener europäischer Forschungseinrichtungen im Bereich der Komplementärmedizin aufbauen und die internationale Kooperation fördern. In verschiedenen Arbeitsgruppen wird man sich unter anderem mit der Terminologie, den rechtlichen Voraussetzungen für die Anwendung naturheilkundlicher Verfahren, mit den Bedürfnissen der Patienten, mit dem Stellenwert dieser Verfahren im Versorgungssystem sowie mit forschungsmethodischen Fragen befassen. Dadurch soll ein umfassendes Bild der aktuellen Situation der Komplementärmedizin in Europa gezeichnet werden, das als Ausgangspunkt für künftige Forschungsaktivitäten dienen soll. Hierzu wird das Projekt CAMbrella am Ende eine Empfehlungsliste erarbeiten.

Die Strategie, nicht ein einzelnes Forschungsprojekt zu fördern, sondern zu sondieren, welche Forschungsthemen der Komplementärmedizin sich in der aktuellen Situation in Europa als die wichtigsten für die nächste Zukunft erweisen (und wie diese Forschungsfragen anzugehen sind), ist bei allseits begrenzten Ressourcen gut nachvollziehbar.
In die Freude mischt sich aber auch Verärgerung. Warum muss dieses Projekt schon an der Startlinie aus den «eigenen Reihen» angegriffen werden? Faire Kritik am inhaltlichen Aufbau oder - wenn es schon so weit wäre - an publizierten Projektergebnissen, sind hier selbstverständlich nicht gemeint. Es ist vielmehr die in die Öffentlichkeit gebrachte Meinung, das hier tätige Forscherkonsortium bestünde ausschließlich aus Befürwortern [1]. Eine Kollegenschelte, die nicht unwidersprochen stehen bleiben kann. Darf man sich so - bei allen persönlichen Verdiensten und der großen Anerkennung, die sich der fragliche Kollege international mit seiner kritisch-wissenschaftlichen Haltung mit Recht erworben hat - als Einziger mit dem Attribut des «Nicht-Befürworters» bzw. «neutralen» Forschers über alle anderen Kolleginnen und Kollegen der komplementärmedizinischen Forschung stellen? Dies ist intellektuell nicht redlich! Schade um die Sache, schade um die Kollegialität!

Wohl im Zuge der derzeitigen Kontroverse um die Homöopathie in Großbritannien wird auch der Vorwurf laut, die Europäische Kommission betreibe mit dem CAMbrellaProjekt lediglich die Förderung einer Lobby-Gruppe [2]. Die Kritik an der Projektausschreibung im Bereich Gesundheit des 7. Rahmenprogramms der EU wird hier gleich auf die Förderpolitik des National Center for Complementary and Alternative Medicine (NCCAM) der Vereinigten Staaten erweitert: Beiden Fällen wird «waste of money» beschieden.

Dennoch sollte die Freude über das Gelungene überwiegen! Ich bin davon überzeugt, dass dieser Start sowohl für uns als Forschende auf diesem Gebiet als auch für die politische Willensbildung in ganz Europa ein wichtiger Schritt ist. Noch vor kurzer Zeit war dieser aus meiner Sicht kaum vorstellbar. In diesem Sinne rufe ich - analog Obamas Wahlruf - mit Stolz: Yes, we CAM!

\section{Literatur}

1 Zoë Corbyn: Alternative medicine plan is 'waste of money'. www.timeshighereducation.co.uk/story.asp? sectioncode $=26 \&$ storycode $=410186$.

2 Gibney E: Complementary medicine research network needs more critical voices. Research Europe, 28 Jan 2010.

\section{KARGER}

Fax +497614520714

Information@Karger.de

www.karger.com (c) 2010 S. Karger GmbH, Freiburg

Accessible online at:

www.karger.com/fok
Prof. Dr. med. Dieter Melchart

Zentrum für naturheilkundliche Forschung

der II. Med. Klinik und Poliklinik, TU München

Kaiserstr. 9, 80801 München, Deutschland

Tel. +49 89 726697-0, Fax -21

Dieter.Melchart@1rz.tu-muenchen.de 